\title{
Negative muscle pennation angle as a sign of massive musculotendinous retraction after tendon tear: paradoxical function of the vastus lateralis muscle
}

\author{
Meyer, D C ; Gerber, C ; Farshad, M
}

\begin{abstract}
Case report: In the presented case, an isolated vastus lateralis tendon tear resulted in a negative pennation angle of the distal muscle fibers acting paradoxical to the physiological direction of the proximal muscle fibers. This observation makes the value of an in situ repair of a far retracted tendon tear with interposition of a graft questionable and emphasizes early over late anatomic surgical restoration of the muscle architecture after tendon tears.
\end{abstract}

DOI: https://doi.org/10.1007/s00167-011-1488-y

Posted at the Zurich Open Repository and Archive, University of Zurich

ZORA URL: https://doi.org/10.5167/uzh-59858

Journal Article

Published Version

Originally published at:

Meyer, D C; Gerber, C; Farshad, M (2011). Negative muscle pennation angle as a sign of massive musculotendinous retraction after tendon tear: paradoxical function of the vastus lateralis muscle. Knee Surgery, Sports Traumatology, Arthroscopy, 19(9):1536-1539.

DOI: https://doi.org/10.1007/s00167-011-1488-y 


\title{
Negative muscle pennation angle as a sign of massive musculotendinous retraction after tendon tear: paradoxical function of the vastus lateralis muscle
}

\author{
D. C. Meyer · C. Gerber · M. Farshad
}

Received: 10 December 2010/ Accepted: 15 March 2011/Published online: 29 March 2011

(C) Springer-Verlag 2011

\begin{abstract}
Case report In the presented case, an isolated vastus lateralis tendon tear resulted in a negative pennation angle of the distal muscle fibers acting paradoxical to the physiological direction of the proximal muscle fibers. This observation makes the value of an in situ repair of a far retracted tendon tear with interposition of a graft questionable and emphasizes early over late anatomic surgical restoration of the muscle architecture after tendon tears.
\end{abstract}

Keywords Tendon tear - Quadriceps · Repair ·

Pennation angle $\cdot$ Heal

\section{Introduction}

Tendon tears are a common injury and may lead to pain and loss of function, with corresponding musculotendinous retraction and atrophy due to disuse. As a consequence of tears of a functional subunit of a muscle, it may intuitively be expected that the affected part of the muscle retracts actively in a physiological fashion until possible scarring or healing of the torn tendon part allows for recovery of at least partial function. Here, we present a case in which gross and paradoxical alteration of the muscle architecture resulted in a hitherto undescribed negative (inverted) muscle fiber pennation angle. These changes make such recovery impossible and may help in understanding and decision making regarding treatment of comparable tendon tears. In pennate muscles, the pennation angle is defined as the angle

D. C. Meyer $(\varangle) \cdot$ C. Gerber · M. Farshad

Balgrist University Hospital, University of Zürich,

Forchstr. 340, 8008 Zürich, Switzerland

e-mail: dominik.meyer@balgrist.ch between the muscle fibers and line of muscle action. With muscle contraction, the tendon moves toward the muscle, and the shortened and thickened fibers become arranged at a larger angle. The force-length relationship changes and might have a potential effect on muscle function [16]. With an increasing pennation angle, the effectiveness of each fiber to pull the tendon in the desired direction decreases with the cosine of that angle and reaches zero at $90^{\circ}$ [12]. Therefore, an angle of $90^{\circ}$ and more seems impossible to develop even with a complete tendon tear, which has to our knowledge not been seen or described before and makes this case so interesting to observe.

\section{Case report}

A 35-year-old construction worker suffered a direct trauma during work from a hammer onto the anterolateral thigh, few centimeters proximal to the patella. At a regional hospital, the clinical diagnosis of a tear of the vastus lateralis portion from the quadriceps femoris tendon was made, with direct avulsion from the patella. An attempt for direct open repair of the tendon using sutures directly at the upper pole of the patella was made within a month. However, despite partial weight bearing, pain with subjectively pain-related weakness persisted and an MRI of both thighs was performed 9 months after the attempted repair (Fig. 1). Here, the clinical diagnosis of a tear of the distal vastus lateralis tendon is confirmed with a strong alteration of the corresponding musculotendinous unit, characterized by a massive increase in the muscle fiber pennation angle within the affected muscle. This pennation angle increase was so far progressed that an inversion of the muscle pennation in the more distal portion of the retracted muscle was present. Thereafter, the patient was 


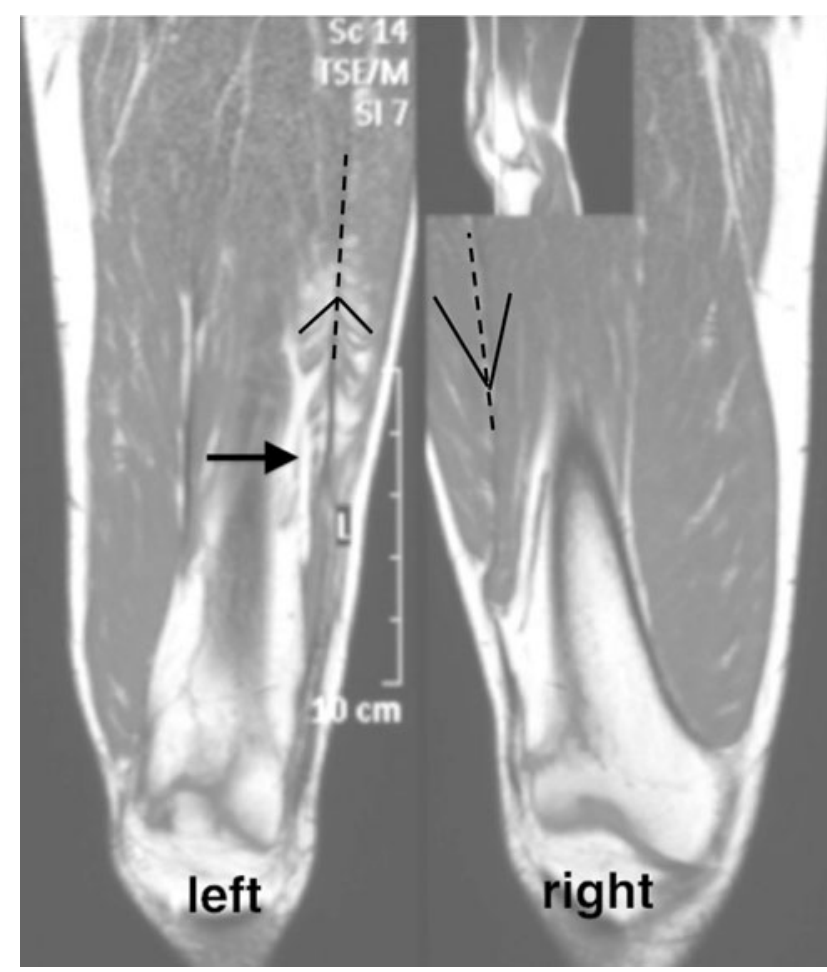

Fig. 1 Frontal MRI sections of both thighs 9 months after trauma and attempted repair, showing the inverted pennation angle of the distal vastus lateralis portion (arrow). (The right side is the intact contralateral side, the line of muscle action is depicted as a dotted line, muscle fiber direction is a full line)

referred to our clinic 1 year later and still complained of persistent pain, subjective cramping of the thigh, and weakness for left knee extension. Clinically, there was a marked atrophy of the quadriceps femoris with, however, full range of motion and no extension lag. We decided to continue with physical therapy and conservative treatment due to the elapsed time since trauma. In the meantime, the patient lost his work and was applying for permanent workers compensation for the described condition, which he received thereafter. Due to continuing pain, an MRI of both thighs was repeated one and a half years after the first MRI (Fig. 2), showing a persistently altered muscle architecture and a $5-\mathrm{cm}$ scar between the retracted tendon end and the patella. The area of altered muscle architecture is $10-21 \mathrm{~cm}$ proximal to the patella. As the patient continued to complain from pain with an objective gap in the vastus lateralis tendon, it was decided to plastically reconstruct the gap with a fascia lata interposition plasty 2 years after the initial trauma. During that operation, it was noted that re-lengthening of the muscle seemed not possible, and a mere in situ repair with filling of the gap was performed using the transplanted fascia lata. Unfortunately, the patient did subjectively not benefit from this intervention regarding pain and limping at 1-year followup and had to continue to receive workers compensation.

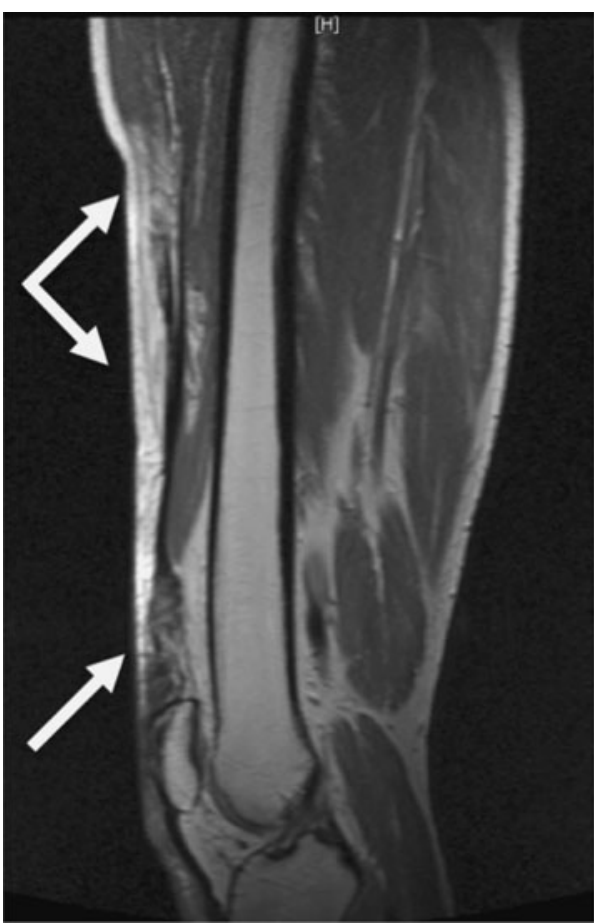

Fig. 2 Sagittal section through the affected thigh one and a half years after the first attempted repair. Distal, there is the scar above the patella with the retracted tendon end (one arrow) and proximal, the still altered musculature (two arrows)

\section{Discussion}

The most important finding of the present case report was the paradoxical alteration of the muscle architecture caused by chronic retraction after tear of the distal vastus lateralis tendon resulting in a hitherto undescribed negative (inverted) muscle fiber pennation angle.

Tendons may extend far into the muscle, where they are termed "central tendon." The central muscle tendon is a typical feature of pennate muscle and is a prerequisite for the synergistic action of the parallel-arranged muscle fibers. With physiological contraction, muscle fibers increase their diameter and shorten. The pennation angle is increased in the contracted muscle, which allows the contracted, shortened, and thickened fibers to find space next to each other $[1,15]$. The opposite effect occurs in hypertrophic muscle such as in bodybuilders, where the hypertrophy of the fibers forces them into an increased pennation angle $[1,11]$ or in atrophied muscle, where the pennation angle is decreased [2]. In case of a chronic tendon tear, however, the fibers suffer from atrophy and shortening (Fig. 3) and with the pathologically increased pennation angle, large gap spaces are opened between the fibers, which are filled with fat and scar tissue [13]. This fat is along with the increased pennation angle considered a secondary sign of long-standing 


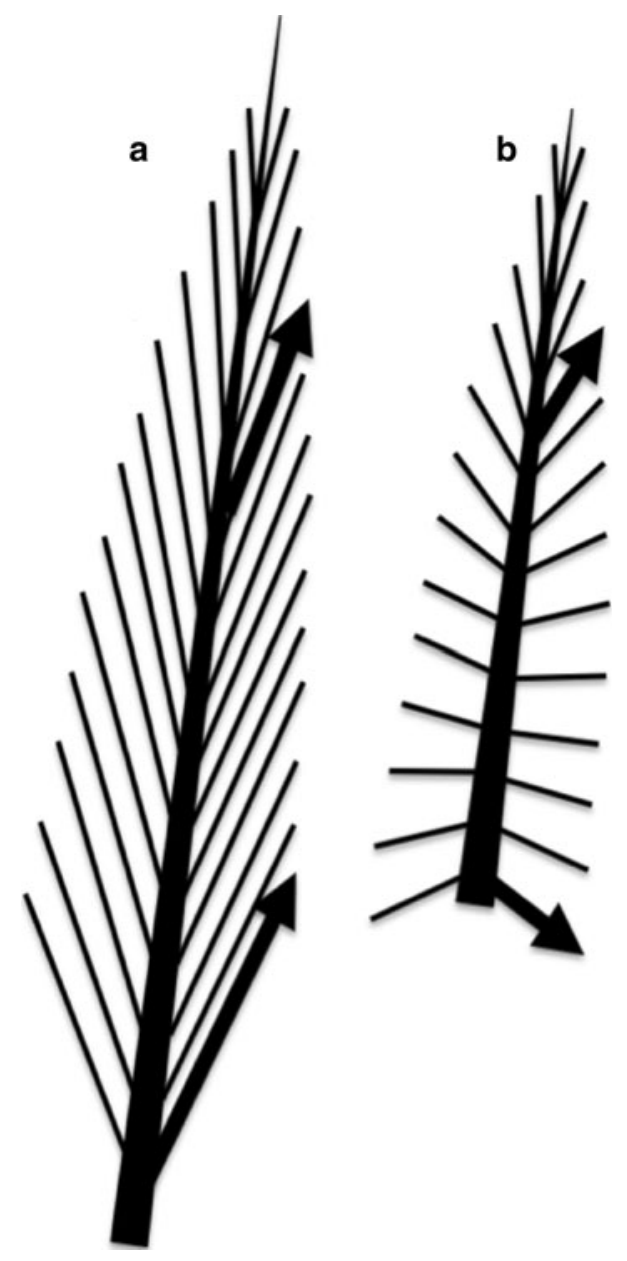

Fig. 3 Distal and proximal muscle fibers synergize physiologically (a), but antagonize pathologically (b) after tendon tear and retraction of the musculotendinous unit. Shortening of the central muscle tendon further supports this paradoxical development

tendon tears $[6,8,10]$. Then, repair may not be possible anymore due to the stiffness and shortening of the musculotendinous unit, which is radiologically and histologically seen as atrophy and fatty infiltration [9, 13]. In a sheep model for chronic tendon tears, it could be shown that proper repair may already be severely compromised 3-6 month after a complete tendon tear [4, 5, 7].

In the presented case, an isolated vastus lateralis tendon tear has occurred. With the massive contraction and chronic shortening of the proximal muscle fibers, the central tendon underwent far more proximal displacement than it could actively have been caused by the local muscle fibers. As the attachment sites of the more distal muscle fibers on bone, fascia, and tendon remain stationary, the ongoing proximalization and most likely accompanying shortening of the central tendon [14] leads to a relative, passive movement of these fiber insertions on the tendon relative to the bone and surrounding muscle fascia by a distance far greater than in active contraction. Here, this results in the observed negative pennation angle. The possible contractile action of the muscle is, of course, paradoxical in this situation; the most proximal fibers will pull into a physiological direction, while the distal fibers pull on the same central tendon into the opposite direction (Fig. 3). To our knowledge, such a seemingly impossible anatomical arrangement of muscle fibers with a negative pennation angle has not been described previously. The conclusion from this geometrical observation is, that following a displacement of the tendon insertion, not only after tear but also for example after a distalization or proximalization of the tibial tuberosity, the more distal muscle fibers are affected in a different way than the proximal fibers. This effect has also been observed in tears of the rotator cuff, where the more distal parts of the rotator cuff muscles suffer radiologically from more intense fatty infiltration than proximal parts [3]. In future studies, it will be interesting to study not only the geometrical but also neurological aspects of this observation, which may contribute to the more distal fatty changes of muscle following tendon tears. The MRI at follow-up shows that also after 1 year, spontaneous rearrangement of the muscle architecture did not occur (Fig. 2).

Clinically, we, therefore, conclude that the value of an in situ repair of a far retracted tendon tear with interposition of a graft may be questionable and that early or late anatomic surgical restoration of the muscle architecture is desirable after tendon tears.

\section{References}

1. Aagaard P, Andersen JL, Dyhre-Poulsen P et al (2001) A mechanism for increased contractile strength of human pennate muscle in response to strength training: changes in muscle architecture. J Physiol 534:613-623

2. Bleakney R, Maffulli N (2002) Ultrasound changes to intramuscular architecture of the quadriceps following intramedullary nailing. J Sports Med Phys Fitness 42:120-125

3. Fabis J, Kordek P, Bogucki A et al (1998) Function of the rabbit supraspinatus muscle after detachment of its tendon from the greater tubercle. Observations up to 6 months. Acta Orthop Scand 69:570-574

4. Gerber C, Meyer DC, Frey E et al (2009) Neer award 2007: reversion of structural muscle changes caused by chronic rotator cuff tears using continuous musculotendinous traction. An experimental study in sheep. J Shoulder Elbow Surg 18:163-171

5. Gerber C, Meyer DC, Schneeberger AG et al (2004) Effect of tendon release and delayed repair on the structure of the muscles of the rotator cuff: an experimental study in sheep. J Bone Joint Surg Am 86-A:1973-1982

6. Gerber C, Schneeberger AG, Hoppeler H et al (2007) Correlation of atrophy and fatty infiltration on strength and integrity of rotator cuff repairs: a study in thirteen patients. J Shoulder Elbow Surg 16:691-696 
7. Gerber C, Schneeberger AG, Perren SM et al (1999) Experimental rotator cuff repair. A preliminary study. J Bone Joint Surg Am 81:1281-1290

8. Goutallier D, Postel JM, Bernageau J et al (1995) Fatty infiltration of disrupted rotator cuff muscles. Rev Rhum Engl Ed $62: 415-422$

9. Goutallier D, Postel JM, Bernageau J et al (1994) Fatty muscle degeneration in cuff ruptures. Pre- and postoperative evaluation by CT scan. Clin Orthop Relat Res 304:78-83

10. Goutallier D, Postel JM, Bernageau J et al (1995) La dégéneresence graisseuse des muscles des coiffes tendineuses rompues de l'épaule. Revue du rhumatisme, Ed francaise 62:439-446

11. Kawakami Y, Abe T, Fukunaga T (1993) Muscle-fiber pennation angles are greater in hypertrophied than in normal muscles. J Appl Physiol 74:2740-2744
12. Maganaris CN (2001) Force-length characteristics of in vivo human skeletal muscle. Acta Physiol Scand 172:279-285

13. Meyer DC, Hoppeler H, von Rechenberg B et al (2004) A pathomechanical concept explains muscle loss and fatty muscular changes following surgical tendon release. J Orthop Res 22: 1004-1007

14. Meyer DC, Lajtai G, von Rechenberg B et al (2006) Tendon retracts more than muscle in experimental chronic tears of the rotator cuff. J Bone Joint Surg Br 88:1533-1538

15. Rutherford OM, Jones DA (1992) Measurement of fibre pennation using ultrasound in the human quadriceps in vivo. Eur J Appl Physiol Occup Physiol 65:433-437

16. Winter SL, Challis JH (2010) The expression of the skeletal muscle force-length relationship in vivo: a simulation study. J Theor Biol 262:634-643 